\title{
Diagnósticos de enfermagem para as etapas do crescimento e desenvolvimento de
} crianças utilizando a CIPE $^{\circledR}$

\author{
Ana Márcia Nóbrega Dantas ${ }^{1}$, Gabriela Lisieux Lima Gomes ${ }^{2}$, \\ Kenya de Lima Silva ${ }^{3}$, Maria Miriam Lima da Nóbrega ${ }^{4}$
}

\footnotetext{
${ }^{1}$ Enfermeira. Discente do Programa de Pós-Graduação em Enfermagem, nível Mestrado, Universidade Federal da Paraíba. João Pessoa, PB, Brasil. E-mail: am_nobrega@hotmail.com.

${ }^{2}$ Enfermeira, Mestre em Enfermagem. Discente do Programa de Pós-Graduação em Enfermagem, nível Doutorado, Universidade Federal da Paraíba. Professora Substituta da Universidade Federal da Paraíba. João Pessoa, PB, Brasil. E-mail: gabylisieux@gmail.com.

${ }^{3}$ Enfermeira; Doutora em Enfermagem Fundamental. Professora Adjunta da Universidade Federal da Paraíba. João Pessoa, PB, Brasil. E-mail: kenya_enf@hotmail.com.

${ }^{4}$ Enfermeira, Doutora em Enfermagem. Professora Titular da Universidade Federal da Paraíba. João Pessoa, PB, Brasil. E-mail: miriam@ccs.ufpb.br.
}

Recebido: 12/05/2015.

Aceito: 02/12/2015.

Publicado: 30/06/2016.

Como citar esse artigo:

Dantas AMN, Gomes GLL, Silva KL, Nóbrega MML. Diagnósticos de enfermagem para as etapas do crescimento e desenvolvimento de crianças utilizando a CIPE ${ }^{\circledR}$. Rev. Eletr. Enf. [Internet]. 2016 [acesso em: ____18:e1165. Disponível em: http://dx.doi.org/10.5216/ree.v18.35524.

\begin{abstract}
RESUMO
Pesquisa exploratória descritiva, cujo objetivo foi construir enunciados de diagnósticos de enfermagem para as fases do crescimento e do desenvolvimento da criança na atenção básica, utilizando-se os termos identificados a partir do Manual do Crescimento e Desenvolvimento do Ministério da Saúde e a Classificação Internacional para a Prática de Enfermagem $\left(\mathrm{CIPE}^{\circledR}\right)$. Foram construídos 111 enunciados de diagnósticos de enfermagem, que foram classificados de acordo com as etapas do desenvolvimento e do crescimento da criança, 23, no período neonatal; 40, na primeira infância; e 48, na fase pré-escolar. Acredita-se que a construção de enunciados de diagnósticos de enfermagem para as fases do crescimento e do desenvolvimento da criança contribuem diretamente para melhorar a qualidade da assistência prestada. Pretende-se proceder à validação clínica dos enunciados de diagnósticos de enfermagem, a fim de possibilitar a integração do conhecimento científico com a prática, na assistência à criança na atenção primária à saúde.
\end{abstract}

Descritores: Diagnóstico de Enfermagem; Criança; Crescimento e Desenvolvimento.

\section{INTRODUÇÃO}

A atenção à saúde da criança é uma área prioritária no que diz respeito ao cuidado à saúde, tendo em vista que é no período da infância que ocorre o desenvolvimento das inúmeras capacidades de um indivíduo, 
assim como também, disfunções no funcionamento do organismo, sendo mais vulnerável nos primeiros anos de vida, podendo acarretar em sequelas na vida $\operatorname{adulta}^{(1)}$.

No que diz respeito à assistência de enfermagem à criança, é fundamental que o enfermeiro realize o processo de maneira organizada e criteriosa para atender às necessidades da criança e de sua família. Assim, a comunicação verbal e a não verbal são ferramentas essenciais para o processo de cuidar. No entanto, inúmeras barreiras dificultam o progresso da comunicação, como a falta de interação nas atividades assistenciais. Por isso, deve-se estabelecer um elo construtivo e compreensivo com a finalidade de amenizar os conflitos e de estimular a criança e sua família a serem confiantes e capazes de mudar o estilo de vida ${ }^{(2)} \mathrm{e}$ garantir a inserção da família como protagonistas do processo de cuidar.

Na perspectiva da atenção primária no Brasil, o cuidado prestado à criança, apresenta como base de sua estrutura à promoção a saúde, prevenção, diagnóstico precoce e recuperação dos agravos infantis, concomitante ao acompanhamento do crescimento e desenvolvimento. Possuindo ações integrantes, caracterizadas pelo controle das doenças prevalentes, incentivo do aleitamento materno, informando sobre as condições alimentares e a importância da imunização(1).

A redução da mortalidade infantil e a expansão da assistência contribuíram para um progressivo aumento na atenção à saúde da criança. Por essa razão, a assistência à saúde infantil ampliou seus horizontes e não incide apenas na perspectiva da doença, é necessário que haja o acompanhamento do seu crescimento e desenvolvimento, principalmente no período de zero a seis anos, para garantir excelência na qualidade de sua vida ${ }^{(3)}$.

As etapas que compreendem o período de seu crescimento e desenvolvimento até os seis anos de idade são denominadas de Período pré-natal, que vai da concepção ao nascimento; Período neonatal, do nascimento até 28 dias; Primeira infância, dos 29 dias aos dois anos de idade; e a fase Pré-escolar, dos dois aos seis anos de vida ${ }^{(4)}$. Considerando as etapas e os diversos aspectos relacionados ao crescimento e ao desenvolvimento da criança, é indispensável que o enfermeiro, como membro da equipe de saúde, saiba avaliar dados referentes ao estado nutricional, ao estado de sono, às curvas de perímetro cefálico, ao peso, ao comprimento, à altura, ao índice de massa corporal por idade e aos marcos do desenvolvimento ${ }^{(5)}$.

Os referidos marcos são observados no período neonatal, quanto ao predomínio do tônus flexor, assimetria postural, preensão reflexa, percepção do rosto da mãe; na primeira infância é verificado no bebê, posição de bruços levantando a cabeça e os ombros, demonstra o sorriso social, apresenta preensão voluntária das mãos, vira a cabeça na direção de uma voz ou objeto sonoro, adquire noção de profundidade, senta-se sem apoio, engatinha, apresenta reações a pessoas estranhas, anda com ou sem apoio, possui acuidade visual de um adulto, corre ou sobe pequenos degraus, diz seu próprio nome, reconhecesse no espelho; e nos marcos da fase pré-escolar é percebido que a criança veste sem auxilio, conta ou inventa pequenas histórias, a memória e a linguagem aumentam, apresenta comportamento egocêntrico, começam a compreender a constância de gênero, os amigos assumem importância ${ }^{(4)}$.

Ainda é imprescindível registrar na Caderneta da Saúde da Criança o calendário de vacinação e 
observar a interação com a família, o lazer, as condições de saúde, as necessidades apresentadas pela criança e as queixas expostas pelos pais. A partir desses parâmetros, pode-se avaliar se crescimento e o desenvolvimento estão dentro da normalidade ou se há risco de déficit no referido processo ${ }^{(5)}$.

Acompanhar o crescimento e o desenvolvimento da criança é indispensável, pois dá subsídios para o estabelecimento de diagnósticos de enfermagem e, por conseguinte, para o planejamento dos cuidados. Tais fatos contribuem diretamente para melhorar a qualidade da assistência prestada, sistematizar a assistência e implementar as etapas do processo de enfermagem, o que agrega valor à qualidade de vida dessas crianças.

A construção de enunciados de diagnósticos para cada etapa do desenvolvimento, é uma ferramenta que irá contribuir significativamente para o processo da assistência às crianças, uma vez que o acompanhamento do crescimento e desenvolvimento são grandes indicadores da sua qualidade de vida ${ }^{(6)}$. Além do mais, a estruturação direcionará as necessidades do cuidado, esclarecendo ao enfermeiro suas atribuições frente à criança, padronizando uma linguagem universal e facilitando a compreensão entre os enfermeiros e outros profissionais de saúde. Ademais, possibilita a documentação de informações de enfermagem, auxiliando o cuidado à criança e oferece maior visibilidade ao conhecimento profissional ${ }^{(7)}$.

Considerando a relevância da implementação do processo de enfermagem frente à criança nas diferentes fases de crescimento e desenvolvimento, este artigo teve como objetivo construir enunciados de diagnósticos de enfermagem por etapas do desenvolvimento e do crescimento da criança.

\section{MÉTODOS}

Pesquisa exploratória e descritiva, realizada no período de fevereiro a abril de 2014 , vinculada ao Grupo de Estudos e Pesquisas sobre Fundamentação da Assistência de Enfermagem (GEPFAE), do Programa de Pós-graduação em Enfermagem, da Universidade Federal da Paraíba (UFPB), com a finalidade de estruturar enunciados de diagnósticos de enfermagem para as fases do crescimento e do desenvolvimento da criança na atenção básica, utilizando-se os termos identificados no Manual do Crescimento e do Desenvolvimento do Ministério da Saúde, quando foram elencados 576 termos que foram submetidos ao processo de mapeamento cruzado, o que resultou em 187 termos constantes e 389 não constantes no Modelo de Sete Eixos da CIPE ${ }^{\circledR} 2011$.

Para a identificação dos termos, foram feitas leituras do Manual e realizado um grifo nos termos, para isolá-los, os quais, posteriormente, foram recortados, utilizando-se um aplicativo de seleção do software Word ${ }^{\circledR}$ for Windows 2007, atentando para a normalização dos termos com a manutenção de termos e/ou expressões de interesse para os objetivos do estudo e a eliminação daqueles considerados ad hoc como não pertinentes. Para tanto, os termos identificados e os termos da $\mathrm{CIPE}^{\circledR}$ foram incluídos em duas planilhas do Excel for Windows, depois, importados para o Programa Access for Windows, para se construir a tabela de termos, que foram submetidos ao processo de mapeamento cruzado, o que culminou com a ligação dos termos identificados nos documentos com os da $\mathrm{CIPE}^{\circledR}$ e a identificação dos constantes e dos não constantes nessa terminologia ${ }^{(8)}$. 
Para compor os enunciados de diagnósticos de enfermagem, utilizaram-se as diretrizes do Conselho Internacional de Enfermeiros (CIE), consubstanciadas na norma ISO 18.104, que preconiza a utilização obrigatória de um termo do eixo Foco e um do eixo Julgamento, que podem ser acrescidos de termos de outros eixos, conforme a necessidade de elucidar o conceito de diagnóstico estruturado ${ }^{(9)}$. Além dessas diretrizes, foram considerados o julgamento clínico e a experiência das autoras na assistência à criança, os quais possibilitaram a análise da hipótese diagnóstica com posterior julgamento quanto a sua adequação à determinada etapa do crescimento e do desenvolvimento e, além disso, quanto à pertinência destas para a prática da assistência.

Em seguida, os enunciados construídos foram classificados por etapa do desenvolvimento e do crescimento da criança: período neonatal, que vai do nascimento até 28 dias; a primeira infância, dos 29 dias aos dois anos de idade; e a fase pré-escolar, dos dois aos seis anos de vida. Esses enunciados foram analisados levando-se em consideração o Manual do Crescimento e do Desenvolvimento do Ministério da Saúde ${ }^{(4)}$.

Como não houve envolvimento de seres humanos na pesquisa, não foi necessário submeter o projeto ao Comitê de Ética em Pesquisa da Instituição.

\section{RESULTADOS}

Foram construídos 111 enunciados de diagnósticos de enfermagem, classificados de acordo com as etapas do crescimento e do desenvolvimento da criança - 23 (20,7\%), no período neonatal; 40 (36,0\%), na primeira infância; e 48 (43,3\%), na fase pré-escolar, os quais estão apresentados nos Quadros 1, 2 e 3.

Quadro 1: Enunciados de diagnósticos de enfermagem elaborados, a partir da $\mathrm{CIPE}^{\circledR}$, para o período neonatal para acompanhar o desenvolvimento e o crescimento da criança. João Pessoa, PB, Brasil, 2014.

\begin{tabular}{|l|l|}
\hline \multicolumn{2}{|l|}{ Enunciados de diagnósticos de enfermagem } \\
\hline 1. Acompanhamento do crescimento e do desenvolvimento completo; & 12. Cólica (especificar o grau); \\
\hline 2. Acompanhamento do crescimento e do desenvolvimento incompleto; & 13. Conduta motora deficiente; \\
\hline 3. Alergia; & 14. Desenvolvimento prejudicado da criança; \\
\hline 4. Amamentação exclusiva; & 15. Padrão de desenvolvimento deficiente; \\
\hline 5. Amamentação interrompida & 16. Padrão de desenvolvimento eficaz; \\
\hline 6. Amamentação positiva; & 17. Resposta eficaz à medicação; \\
\hline 7. Audição deficiente (diminuída); & 18. Risco de condições patológicas; \\
\hline 8. Ausência de choro; & 19. Sono adequado; \\
\hline 9. Calendário vacinal completo; & 20. Sono prejudicado; \\
\hline 10. Calendário vacinal incompleto; & 21. Sucção deficiente; \\
\hline 11. Choro (especificar o grau). & 22. Sucção normal; \\
\hline & 23. Visão deficiente. \\
\hline
\end{tabular}


Quadro 2: Enunciados de diagnósticos de enfermagem elaborados, a partir da $\mathrm{CIPE}^{\circledR}$, para o período da primeira infância (29 dias a dois anos) para acompanhar o desenvolvimento e o crescimento da criança. João Pessoa, PB, Brasil, 2014.

\section{Enunciados de diagnósticos de enfermagem}

1. Acompanhamento do crescimento e do desenvolvimento incompleto;

2. Acompanhamento do crescimento e do desenvolvimento completo;

3. Agitação (especificar o grau);

4. Alergia;

5. Amamentação exclusiva;

6. Amamentação interrompida;

7. Amamentação positiva;

8. Atraso no crescimento;

9. Audição deficiente;

10. Calendário vacinal completo;

11. Calendário vacinal incompleto;

12. Capacidade de brincar em grupo;

13. Capacidade de dizer o próprio nome;

14. Capacidade de levar os alimentos à boca;

15. Capacidade de reconhecer as cores;

16. Choro (especificar o grau);

17. Cólica (especificar o grau);

18. Crescimento eficaz;

19. Crescimento físico deficiente.

20. Crescimento físico normal;
21. Crescimento retardado;

22. Desenvolvimento emocional normal;

23. Desenvolvimento emocional prejudicado;

24. Desenvolvimento psicomotor normal;

25. Desenvolvimento psicomotor prejudicado;

26. Febre (hipertermia)

27. Hipotermia;

28. Irritabilidade (especificar o grau);

29. Padrão de desenvolvimento eficaz;

30. Padrão de desenvolvimento prejudicado;

31. Percepção alterada;

32. Percepção sensorial eficaz;

33. Percepção visual deficiente;

34. Resposta eficaz à medicação;

35. Resposta eficaz à nutrição enteral;

36. Risco de agitação;

37. Risco de febre;

38. Risco de queda;

39. Termorregulação ineficaz;

40. Termorregulação normal.

Quadro 3: Enunciados de diagnósticos de enfermagem elaborados, a partir da $\mathrm{CIPE}^{\circledR}$, para a fase pré-escolar para acompanhar o desenvolvimento e o crescimento da criança. João Pessoa, PB, Brasil, 2014.

\section{Enunciados de diagnósticos de enfermagem}

1. Acompanhamento do crescimento e do desenvolvimento completo;

2. Acompanhamento do crescimento e do desenvolvimento incompleto;

3. Agitação (especificar o grau);

4. Alergia;

5. Bem-estar emocional;

6. Calendário vacinal completo;

7. Calendário vacinal incompleto;

8. Capacidade de brincar em grupo;

9. Capacidade de dizer o próprio nome;

10. Capacidade de levar os alimentos à boca;

11. Capacidade de reconhecer as cores;

12. Atenção deficiente (déficit de atenção);

13. Audição deficiente;

14. Capacidade de se vestir;

15. Comunicação verbal eficaz;

16. Comunicação verbal prejudicada

17. Conhecimento adequado da mãe (especificar);

18. Conhecimento deficiente da mãe;

19. Crescimento físico deficiente;

20. Crescimento físico normal.

21. Deficiência no funcionamento psíquico;

22. Desenvolvimento emocional deficiente;

23. Desenvolvimento emocional normal;

24. Desenvolvimento infantil dependente;
25. Dor;

26. Dor aguda;

27. Dor ausente;

28. Dor crônica;

29. Dor melhorada;

30. Desenvolvimento prejudicado;

31. Desenvolvimento psicomotor deficiente;

32. Desenvolvimento psicomotor normal;

33. Febre (hipertermia);

34. Hipotermia;

35. Irritabilidade (especificar o grau);

36. Isolamento social;

37. Olfato eficaz;

38. Padrão de desenvolvimento completo;

39. Padrão de desenvolvimento incompleto; 40. Resposta eficaz à medicação;

41. Resposta eficaz à nutrição enteral;

42. Risco de agitação;

43. Risco de febre;

44. Risco de lesão;

45. Termorregulação ineficaz;

46. Termorregulação normal;

47. Vômito;

48. Vômito ausente. 


\section{DISCUSSÃO}

O crescimento e o desenvolvimento infantil são delimitados e variam conforme o referencial teórico. São apresentados pela literatura como fenômenos distintos que, invariavelmente, exercem influências sobre o outro. São processos que iniciam no momento da concepção e perduram por todo o período de maturação física, cognitiva e social da criança, caraterizado por seu dinamismo frente às alterações pertinentes a cada etapa que se relaciona com os períodos etários ${ }^{(8)}$.

Assim, a estruturação de enunciados de diagnósticos de enfermagem é relevante no sentido de nortear a assistência de enfermagem. Sua identificação é impulsionada pelo julgamento diagnóstico, que auxilia o enfermeiro a planejar suas ações e, por conseguinte, a implementar suas atividades e avaliar o plano de cuidados prestado.

Os 23 enunciados de diagnósticos de enfermagem estruturados para o período neonatal, que se estende do nascimento até o 28 o dia de vida da criança, refletem o acelerado e imaturo processo de desenvolvimento do neonato, associado as suas necessidades nutricionais. Sob outra ótica, identificaram-se alterações relacionadas ao período puerperal da mãe, como a amamentação, por exemplo.

Nessa etapa, o olhar do profissional auxilia a família a compreender bem mais o crescimento e o desenvolvimento da criança, a perceber e minimizar possíveis alterações, uma vez que alterações evidenciadas e não tratadas no período neonatal poderão desencadear problemas futuros. Em vista disso, o profissional deve ser capaz de fazer uma avaliação criteriosa e diagnósticos específicos para a faixa etária que contribuam para uma assistência de boa qualidade, visando à promoção, à prevenção e à reabilitação da saúde.

$\mathrm{Na}$ primeira consulta do neonato, as principais finalidades são de identificar problemas de amamentação e higienização da criança, imunização e da triagem neonatal, que é um conjunto de exames que podem revelar patologias de caráter metabólico, genético e infeccioso, cujos sintomas são, na maioria dos casos, imperceptíveis nesse período ${ }^{(10)}$. Tais exames concernem ao teste do pezinho, da orelhinha, do ouvido e do coração. Esse é um momento imprescindível para identificar os diagnósticos de enfermagem que irão guiar o plano assistencial e focar em avaliações subsequentes.

O acompanhamento do perímetro cefálico, um fator sobremaneira importante, deverá ser observado nas consultas de enfermagem, já que, por meio desse acompanhamento, é possível observar se o aumento cefálico está dentro dos parâmetros de normalidade ou não. Um dos aspectos que favorece o crescimento adequado do perímetro cefálico é o fator nutricional, a amamentação dispõe de nutrientes, como o ferro e os ácidos graxos poli-insaturados que são elementos diferenciais beneficiando este crescimento. Nas crianças amamentadas, em longo prazo, o crescimento do perímetro cefálico está relacionado a um melhor desenvolvimento mental e desenvolvimento psicomotor ${ }^{(11)}$.

A estratégia do aleitamento materno deve ser exclusiva nos seis primeiros meses e, até a primeira infância, é aconselhável um complemento alimentar, de acordo com a idade da criança. Inúmeros são os benefícios da amamentação, devido ao elevado valor nutricional que pode conferir efeito protetor contra 
infecções, alergias, desordens metabólicas, entre outros. Assim, compete ao enfermeiro adotar condutas favoráveis à amamentação, verificando a sucção, a deglutição, a respiração, o estado da mama e, se necessário, intervir quando a lactação não estiver eficiente. Ademais, deve orientar a puérpera sobre a importância do aleitamento, retirar suas dúvidas e deixá-la segura para amamentar ${ }^{(12)}$.

Para a primeira infância, foram construídos 40 enunciados de diagnósticos de enfermagem, que guardam uma relação direta com os avanços do crescimento e do desenvolvimento infantil, uma vez que é nesse período em que acontecem as maiores e mais rápidas modificações na criança, principalmente no tocante ao domínio neuropsicomotor.

O desenvolvimento neurológico estimula as mais diversas percepções e habilidades ${ }^{(13)}$. Posto isso, a criança inicia um processo de independência, quando começa a engatinhar, a sentar, a andar ${ }^{(14)}$, a reconhecer pessoas, objetos e cores, sorrir, brincar em grupo, pronunciar palavras, entre outros. Em vista disso, a maturidade psicomotora influenciará no crescimento e nas habilidades motoras e psicossociais, no domínio e na aquisição da fala. Fatores biológicos e ambientais podem alterar o processo de crescimento e desenvolvimento infantil ${ }^{(15)}$.

Dentre os determinantes biológicos, revelam-se como possíveis causas do atraso do desenvolvimento das crianças alterações emocionais, de atenção, do estado comportamental, notadas a partir dos seis meses de vida ${ }^{(16)}$. Ademais, questões genéticas e hormonais também podem contribuir para alterar o crescimento e o desenvolvimento delas. No que concerne ao ambiente, podem-se relacionar questões socioeconômicas e a crença da família ${ }^{(15)}$.

O olhar do enfermeiro frente ao ritmo de mudanças é um fator substancial que contribui para o crescimento e o desenvolvimento eficaz na primeira infância. Para isso, o profissional de Enfermagem deverá atender à criança de forma global e estar atento aos sinais verbais e não verbais apresentados pela criança e seu acompanhante, procurando investigar e minimizar as alterações em tempo hábil.

Para a fase pré-escolar, foram estruturados 48 enunciados de diagnósticos de enfermagem. A maior porcentagem nessa etapa relaciona-se à maior exposição da criança ao meio social, uma vez que suas habilidades estão em processo de aprimoramento. Por conseguinte, a linguagem apresenta-se como uma das mais importantes aquisições nesse período. Mediante essa estimulação, a capacidade de compreender e de raciocinar começa a ser ampliada e aumenta cada vez mais seus conhecimentos. Além disso, favorece o desenvolvimento da motricidade e a interação da criança com o meio ${ }^{(17)}$. Um dos fatores que a impossibilitam de inter-relacionar é o distúrbio auditivo, por não conseguir assimilar o que acontece em sua volta ${ }^{(18)}$.

No que concerne à maturidade cognitiva, constata-se que a brincadeira, na infância, promove a interação com outras crianças e adultos, a memorização, a imitação, e assimilação do que é imaginário e do que é real ${ }^{(17)}$.

A alergia é fator importante a ser destacado na assistência às crianças, porquanto pode estar relacionada a diversas causas, como medicamentos e alimentos, por exemplo, a última causa importante de alteração no crescimento e no desenvolvimento infantil. A alergia ao leite de vaca tanto pode provocar 
alterações gastrointestinais, como diarreia e perda de peso, quanto respiratória, cutânea e risco de choque anafilático ${ }^{(19)}$.

A imunização na infância é um fator expressivo para prevenir doenças e promover saúde. Quando agregada ao processo de desenvolvimento e crescimento infantil, a vacinação tende a se revelar mais eficiente ${ }^{(20)}$. Apesar de gratuita e benéfica, estima-se que milhares de crianças ainda morrem por doenças imunopreveníveis ${ }^{(21)}$, devido à falta de conhecimento sobre a importância da imunização, associada à aflição dos pais diante do procedimento doloroso ${ }^{(20)}$ ou de receio frente aos eventos adversos.

Nessa perspectiva, cabe ao enfermeiro adotar estratégias para orientar o cuidador sobre a imunização. Uma boa relação com os pais ${ }^{(20)}$ e a criança pode contribuir para efetivar essas ações. Realizar busca ativa das crianças que estão com calendário vacinal incompleto e fazer palestras com as gestantes sobre a importância da vacinação, do nascimento ao longo da infância, são medidas que precisam ser realizadas constantemente nas unidades básicas de saúde.

O estudo constatou o quantitativo de enunciados de diagnósticos de enfermagem que poderão ser utilizados de forma padronizada para melhorar as ações de cuidado durante as consultas das crianças de zero a seis anos de idade para acompanhar seu crescimento e desenvolvimento. Embora exista uma estratégia padronizada para acompanhar o desenvolvimento delas, a consulta de enfermagem deve ser feita de modo que o profissional proceda ao processo de enfermagem, e os diagnósticos são fundamentais para a realização de intervenções focadas em problemas específicos e parâmetro para avaliações nas consultas seguintes. Ademais, há evidências de que é difícil perceber os fatores de risco para o desenvolvimento e o crescimento infantil. Esse fato decorre, na maioria das vezes, pela não abordagem desse tema pelos profissionais na atenção primária da saúde ${ }^{(22)}$.

\section{CONCLUSÃO}

A utilização do processo de enfermagem na prática profissional favorece o estabelecimento do vínculo entre o enfermeiro, a criança e a mãe e propicia a identificação de enunciados de diagnósticos que, juntos com a implementação da assistência, culminam com a qualificação do trabalho profissional.

A construção de enunciados de diagnósticos de enfermagem por etapas do crescimento e do desenvolvimento da criança, viabilizados mediante o cruzamento de termos identificados no Manual do Crescimento e Desenvolvimento do Ministério da Saúde com a Classificação Internacional para a Prática de Enfermagem ( $\mathrm{CIPE}^{\circ}$ ), pode contribuir com o profissional de Enfermagem na assistência à criança e no estabelecimento de uma relação de ajuda mútua para a família, no processo de crescimento e desenvolvimento infantil, na perspectiva de melhorar a qualidade de sua vida.

No contexto da assistência à criança e da especificidade do cuidado, é necessário elaborar diagnósticos de enfermagem também para a família, especialmente na figura da genitora. Nesse sentido, destacam-se as limitações desta pesquisa no tocante à relevância da identificação de enunciados de diagnósticos de enfermagem para a família, considerando, sobretudo, a indissolubilidade do binômio mãe e filho. 
O desenvolvimento de outros estudos é recomendado, com o objetivo de estruturar enunciados de diagnósticos de enfermagem para crianças em suas diferentes fases de crescimento e desenvolvimento, e para sua família, considerando a utilização de outras literaturas pertinentes à área da assistência infantil. A posteriori, objetiva-se proceder à validação clínica dos enunciados de diagnósticos de enfermagem levantadas neste estudo, a fim de possibilitar a integração do conhecimento científico com o prático, assim como a utilização de uma linguagem unificada para a documentação da prática de enfermagem na assistência à criança na atenção primária à saúde.

\section{REFERÊNCIAS}

1. Gubert FA, Santos DAS, Pinheiro MTM, Brito LLMS, Pinheiro SRCS, Martins MC. Development of a Nursing protocol for childcare consultations. Rev Rene [Internet]. 2015 [acesso em: 30 jun. 2016];16(1):81-9. Disponível em: http://dx.doi.org/10.15253/2175-6783.2015000100011.

2. Martinez EA, Tocantins FR, Souza SR. The specificities of communication in child nursing care. Rev Gaucha Enferm [Internet]. 2013 [acesso em: 30 jun. 2016];34(1):37-44. Disponível em: http://dx.doi.org/10.1590/S198314472013000100005 .

3. Falbo BCP, Andrade RD, Furtado MCC, Mello DF. Estímulo ao desenvolvimento infantil: produção do conhecimento em enfermagem. Rev Bras Enferm [Internet]. 2012 [acesso em: 30 jun. 2016];65(1):148-54. Disponível em:

http://dx.doi.org/10.1590/\$0034-71672012000100022.

4. Ministério da Saúde. Saúde da criança: crescimento e desenvolvimento [Internet]. Brasília: Ministério da Saúde; 2012 [acesso em: 30 jun. 2016]. Disponível em: http://189.28.128.100/dab/docs/publicacoes/cadernos_ab/caderno_33.pdf.

5. Moreira M, Gaíva M. Monitoring of child growth and development: analysis of records of nursing consultations. Rev Pesqui Cuid é Fundam Online [Internet]. 2013 [acesso em: 30 jun. 2016];5(2):3757-66. Disponível em:

http://dx.doi.org/10.9789/2175-5361.2013v5n2p3757.

6. Martins CBG, Pessoa TAO, Lima FCA, Gaíva MAM. Crescimento e desenvolvimento de recém-nascidos de risco em Cuiabá: inquérito domiciliar. Saúde (Santa Maria) [Internet]. 2014 [acesso em: 30 jun. 2016];40(2):155-62. Disponível em: http://dx.doi.org/10.5902/2236583413345.

7. Luciano TS, Nóbrega MML, Saparolli ECL, Barros ALBL. Cross mapping of nursing diagnoses in infant health using the international classification of nursing practice. Rev Esc Enferm USP [Internet]. 2014 [acesso em: 30 jun.

2016];48(2):250-6. Disponível em: http://dx.doi.org/10.1590/\$0080-623420140000200008.

8. Dantas AMN, Souza GLL, Nóbrega MML. Mapeamento de termos da prática de enfermagem no acompanhamento do crescimento e desenvolvimento da criança. Enferm. Foco [Internet]. 2013 [acesso em: 30 jun. 2016];4(2):92-6.

Disponível em: http://revista.portalcofen.gov.br/index.php/enfermagem/article/view/533.

9. International Council Nursing. International classification for nursing practice: version 1.0. Geneva: ICN; 2005. 10. Mendes LC, Santos TT, Bringel FA. Evolução do programa de triagem neonatal no estado do Tocantins. Arq Bras Endocrinol Metabol [Internet]. 2013 [acesso em: 30 jun. 2016];57(2):112-9. Disponível em: http://dx.doi.org/10.1590/S0004-27302013000200003.

11. Jaldin MGM, Pinheiro FS, Santos AM, Muniz NC, Brito LMO. Crescimento do perímetro cefálico nos primeiros seis meses em crianças em aleitamento materno exclusivo. Rev Paul Pediatr [Internet]. 2011 [acesso em: 30 jun.

2016];29(4):509-14. Disponível em: http://ref.scielo.org/7656dt.

12. Baptista SS, Alves VH, Souza RMP, Rodrigues DP, Barbosa MTSR, Vargas GS. Lactação em mulheres com bebês prematuros: reconstruindo a assistência de enfermagem. Rev Pesqui Cuid é Fundam Online [Internet]. 2014 [acesso em: 30 jun. 2016];6(3):1036-46. Disponível em:

http://www.seer.unirio.br/index.php/cuidadofundamental/article/view/3205.

13. Estill DA. Mordidas na primeira infância. Pediatr. mod [Internet]; 2013 [acesso em: 30 jun. 2016]; 49(5):192-6.

Disponível em: http://www.moreirajr.com.br/revistas.asp?fase=r003\&id materia=5383.

14. Formiga CKMR, Cezar MEN, Linhares MBM. Avaliação longitudinal do desenvolvimento motor e da habilidade de 
sentar em crianças nascidas prematuras. Fisioter. Pesqui. [Internet]. 2010 [acesso em: 30 jun. 2016];17(2):102-7. Disponível em: http://dx.doi.org/10.1590/S1809-29502010000200002.

15. Eickmann SH, Malkes NFA, Lima MC. Psychomotor development of preterm infants aged 6 to 12 months. Sao Paulo Med J [Internet]. 2012 [acesso em: 30 jun. 2016];130(5):299-306. Disponível em:

http://dx.doi.org/10.1590/S1516-31802012000500006.

16. Ribeiro DG, Perosa GB, Padovani FH. Fatores de risco para o desenvolvimento de crianças atendidas em Unidades de Saúde da Família, ao final do primeiro ano de vidaaspectos sociodemográficos e de saúde mental materna. Cien Saude Colet [Internet]. 2014 [acesso em: 30 jun. 2016];19(1):215-26. Disponível em: http://dx.doi.org/10.1590/141381232014191.1904.

17. Lopes RCS, Vivian AG, Oliveira DS, Deluchi M, Tudge J, Piccinini CA. Sentimentos maternos frente ao desenvolvimento da criança entre 24 e 28 meses. Estud. psicol. (Campinas) [Internet]. 2012 [acesso em: 30 jun. 2016];29 supl. 1:737-49. Disponível em: http://dx.doi.org/10.1590/s0103-166X2012000500010.

18. Tabaquim MLM, Nardi CGA, Ferrari JB, Moretti CN, Yamada MO, Bevilacqua MC. Avaliação do desenvolvimento cognitivo e afetivo-social de crianças com perda auditiva. Rev CEFAC [Internet]. 2013 [acesso em: 30 jun. 2016];15(6):1475-81. Disponível em: http://ref.scielo.org/t53nqk.

19. Solé D, Amancio OMS, Jacob CMA., Cocco RR, Sarni ROS, Suano F, et al. Guia Prático Guia prático de diagnóstico e tratamento da Alergia às Proteínas do Leite de Vaca mediada pela imunoglobulina E. Rev bras alerg imunopatol [Internet]. 2012 [acesso em: 30 jun. 2016];35(6):203-33. Disponível em: http://www.sbai.org.br/revistas/vol356/Guia35-6.pdf.

20. Figueiredo GLA, Pina JC, Tonete VLP, Lima RAG, Mello DF. Experiences of families in the immunization of Brazilian children under two years old. Rev Lat Am Enfermagem [Internet]. 2011 [acesso em: 30 jun. 2016];19(3):598-605. Disponível em: http://dx.doi.org/10.1590/S0104-11692011000300020.

21. Pugliesi MV, Tura LFR, Andreazzi MFS. Mães e vacinação das crianças: estudo de representações sociais em serviço público de saúde. Rev Bras Saúde Matern Infant [Internet]. 2010 [acesso em: 30 jun. 2016];10(1):75-84. Disponível em: http://dx.doi.org/10.1590/S1519-38292010000100008.

22. Zeppone SC, Volpon LC, Del Ciampo LA. Monitoring of child development held in Brazil. Rev Paul Pediatr [Internet]. 2012 [acesso em: 30 jun. 2016];30(4):594-9. Disponível em: http://dx.doi.org/10.1590/S0103-05822012000400019. 